\title{
Intra-layer flow in fouling layer on membranes
}

\author{
Z. Yang ${ }^{a}$, X.F. Peng ${ }^{a}$, Ming-Yuan Chen ${ }^{b}$, Duu-Jong Lee ${ }^{b}, *$ J.Y. Lai ${ }^{c}$ \\ ${ }^{a}$ Department of Thermal Engineering, Tsinghua University, Beijing 100084, China \\ ${ }^{\mathrm{b}}$ Chemical Engineering Department, National Taiwan University, Taipei 106, Taiwan \\ ${ }^{c}$ Department of Chemical Engineering, R\&D Center of Membrane Technology, Chung Yuan Christian University, Chungli 32023, Taiwan
}

Received 27 September 2006; received in revised form 23 October 2006; accepted 28 October 2006

Available online 12 November 2006

\begin{abstract}
The structure of fouling layer determines the pressure drop across the fouling layer. Three-dimensional distributions of nucleic acids, proteins, $\alpha$-D-glucopyranose polysaccharides, $\beta$-D-glucopyranose polysaccharides and lipids in the biofouling layer that is formed on a mixed cellulose ester membrane were determined using a six-fold staining protocol combined with confocal laser scanning microscopy (CLSM). Based on the three-dimensional volumetric grid model of the fouling layer structure observed from the series of CLSM images, the intra-layer flow field during filtration was simulated using commercial software. The effective permeability of the fouling layer was estimated to be $2.65 \times 10^{-12} \mathrm{~m}^{2}$, which determines the upper estimate on the permeability of the fouling layer. The pores were categorized according to their diameters, using the maximum convex perimeter approach, and then the effects of the blocking pores on the permeability of the fouling layer were investigated. Blocking the large pores that accounted for $15 \%$ of the porosity reduced the mean permeability by $58 \%$.
\end{abstract}

(c) 2006 Elsevier B.V. All rights reserved.

Keywords: Fouling layer; Structure; Staining; CLSM; Modeling; Pore blockage

\section{Introduction}

Membrane fouling reduces the flux and increases the pressure drop across the membrane, causing operational problems and even membrane failure [1]. Le-Clech et al. [2] comprehensively reviewed the literature on membrane fouling. Extracellular polymeric substances (EPSs) have been identified as the principal foulants in membrane bioreactor (MBR) processes [3-11]. Rojas et al. [12] identified a 10-fold increase in the specific resistance of membranes as the protein concentrations are increased from 30 to $100 \mathrm{mg} \mathrm{l}^{-1}$. Lee et al. [13] determined that the supernatant was responsible for up to $37 \%$ of the total resistance during membrane filtration. However, Bouhabila et al. [14] demonstrated that the total resistance to filtration by the supernatant was 76\%. Wisniewski and Grasmick [15] attributed roughly half of the total resistance to filtration to soluble microbial products (SMP) in the supernatant. Lee et al. [16] stated that attached cells and SMPs generated a dynamic membrane on the surface of filter membrane. Cho et al. [17] noted that membrane foul-

\footnotetext{
* Corresponding author. Tel.: +88622362 5632

E-mail address: djlee@ntu.edu.tw (D.-J. Lee).
}

ing was substantial when the extracted amount of EPS ranged 20-80 $\mathrm{mg} \mathrm{g}^{-1}$ MVLSS.

The structure of a thin, deposited layer on a filter membrane has been modeled as a homogeneous porous medium with fixed characteristics [18,19]. High performance size exclusion chromatography (HPSEC) has been used to characterize the EPS extracted from various treatment plants, revealing proteins with $M_{\mathrm{W}} 45-670 \mathrm{kDa}$ and carbohydrates with $M_{\mathrm{W}} 0.5-1 \mathrm{kDa}$ [20,21]. Nagaoka and Nemoto [22] found that EPS with a molecular weight of over $1000 \mathrm{kDa}$ was responsible for most of the fouling of the membrane. However, the carbohydrate level in SMP is correlated with the fouling potential of the membranes [23-26].

Fluorescently labeled stains were employed to analyze the cells and EPS distributions in biofilms [27-29]. Lee et al. [30] probed the cells, polysaccharides and proteins in the biofilm using the following three stains; SYBR Green I, fluorescently labeled lectins and Hoechst 2495. Chen et al. [31] was the first to profile the distributions of proteins, $\alpha$-D-glucopyranose polysaccharides and $\beta$-D-glucopyranose polysaccharides in a fouling layer on a filter membrane. A later study elucidated the structure of the biofouling layer using a quadruple staining scheme (nucleic acids, proteins, $\alpha$ - and $\beta$-D-glucopyranose polysaccha- 
rides) [32] by a series of confocal laser scanning microscopy (CLSM) images of the fouling layer that was converted to bileveled images, from which three-dimensional volumetric grid models of the structure of the fouling layer were generated.

Membrane fouling is a common phenomenon in membrane separation processes. The present work employed a novel sixcolor staining procedure to probe the distributions of proteins, cells (total and dead), $\alpha$ - and $\beta$-D-glucopyranose polysaccharides and lipids in a fouling layer on a membrane surface. The three-dimensional volumetric grid model thus developed was utilized to study the intra-layer flow field in the biological fouling layer using commercial software. The effects of pore blockage on the permeability of the fouling layer were simulated using the detailed structural model. The purpose of this work was to demonstrate a potentially useful tool for building up a structural model of biofouling layer on membrane and for realizing the yielded intra-layer flow field and cross-layer pressure drop. This scheme can be applied on, but is not limited to, study of biofouling in a running MBR.

\section{Experimental}

\subsection{Sample}

Waste activated sludge was obtained from a wastewater treatment plant owned by Presidential Enterprise Corp., Taiwan, which treats $250 \mathrm{~m}^{3}$ of food-processing wastewater daily using primary, secondary and tertiary treatments. The sludge $\mathrm{pH}$ was approximately 6.84 . The chemical oxygen demand (COD) of the sludge and filtrate passing through $0.45 \mu \mathrm{m}$ membrane was $16,000 \mathrm{mg}^{-1}$ (TCOD) and $86.7 \mathrm{mg}^{-1}$ (SCOD), respectively, as determined using a spectrometer (DR/2000, HACH, USA). The elemental composition of the dried samples, determined by an elemental analyzer (Perkin-Elmer $2400 \mathrm{CHN}$ ) was C, 41.3\%; $\mathrm{H}, 6.6 \%$;, $5.4 \%$.

The sludge supernatant obtained after $30 \mathrm{~min}$ of settling was used as the test sample. Flocs were removed to determine the contribution of the colloidal fractions in the supernatant to resistance of the fouling layer. Dead-end membrane filtration tests of obtained supernatant samples were performed in a $30 \mathrm{cmHg}$ vacuum using a $0.45 \mu \mathrm{m}$ mixed cellulose and ester membrane (Advantec MFS, Inc., CA, USA). The membrane and fouling layer were carefully removed from the filtration chamber under fully hydrated conditions for further processing.

\subsection{Staining and imaging}

Calcofluor white was purchased from Sigma (St. Louis, USA). FITC, Con A conjugated with tetramethylrhodamine, SYTO 63, SYTOX blue and Nile red were from Molecular Probes (Carlsbad, CA, USA).

During staining, SYTO $63(20 \mu \mathrm{M})$ was initially dipped on the sample (10 drops) and placed on a shaker table for $30 \mathrm{~min}$. Then, $0.1 \mathrm{M}$ sodium bicarbonate buffer $(\mathrm{pH} 8.8)$ was added to the sample to maintain the amine group in non-protonated form. Then, the FITC solution $\left(10 \mathrm{gl}^{-1}\right)$ was added to the sample (10 drops) for $1 \mathrm{~h}$ at room temperature. Subsequently, the
Con A solution ( $250 \mathrm{mg} \mathrm{l}^{-1}, 10$ drops) was added to the sample and incubated for another $30 \mathrm{~min}$. Calcofluor white (fluorescent brightener $28,300 \mathrm{mg} \mathrm{l}^{-1}, 10$ drops) was then adopted to stain the $\beta$-linked D-glucopyranose polysaccharides for $30 \mathrm{~min}$. In the next step, the Nile Red solution (10 $\mathrm{mg}^{-1}, 10$ drops) was applied to the sample for $10 \mathrm{~min}$. After each of the aforementioned five staining stages, the stained sample was washed twice to remove extra stain using phosphate-buffered saline (PBS) at $\mathrm{pH}$ 7.4. The stained sample was stored at $4{ }^{\circ} \mathrm{C}$. Before observation, the SYTOX Blue solution was added to the samples (10 drops) for incubation for $5 \mathrm{~min}$.

The stained samples were embedded for cryosectioning and frozen at $-20^{\circ} \mathrm{C}$, after which $60 \mu \mathrm{m}$ sections were cut on a cryomicrotome and mounted on the microscopic slides for observation.

Confocal laser scanning microscopy (CLSM; Leica TCS SP2 Confocal Spectral Microscope Imaging System, Germany) was employed to probe the internal structure of fouling layer. The layer was imaged using a $10 \times$ or $20 \times$ objective and analyzed using Leika confocal software. The fluorescence of SYTO 63 was detected by excitation at $633 \mathrm{~nm}$ and emission at $650-760 \mathrm{~nm}$ (red). Excitation at $543 \mathrm{~nm}$ and emission at $550-590 \mathrm{~nm}$ (light blue) were utilized to detect Con A conjugates. Nile Red was excited at $514 \mathrm{~nm}$ and emission collected over $630-700 \mathrm{~nm}$ (yellow). The FITC probe was detected by excitation at $488 \mathrm{~nm}$ and emission at 500-540 $\mathrm{nm}$ (green). The fluorescent intensity of SYTOX Blue was detected by excitation at $458 \mathrm{~nm}$ and emission at $460-500 \mathrm{~nm}$ (purple). The fluorescence of calcofluor white was determined by excitation at $405 \mathrm{~nm}$ and the emission width at $410-480 \mathrm{~nm}$ (blue). Both SYTO 63 and FITC were detected together and the others were detected individually.

\section{Results and discussion}

\subsection{CLSM images of fouling layer}

Fig. 1 presents CLSM images scanned at $5 \mu \mathrm{m}$ above the membrane surface, representing the deposited layer on the membrane surface. In this scanned image, all six probed EPS were distributed in clusters over the fouling layer. The $\beta$-Dglucopyranose polysaccharides, lipids and proteins appeared to correspond closely to the cells (particularly the dead cells), revealing that these EPS may be mostly bound EPS to cell membranes. The presence of $\alpha$-D-glucopyranose polysaccharides however is more weakly correlated with the cells, as noted for the other probed EPS, suggesting that it may contribute to the free EPS that is not tightly bound to the cell membrane.

Fig. 2 displays the side-view images of CLSM, which combine three EPS (Calcofluor white, FITC and SYTO 63), underneath which is a $35 \mu \mathrm{m}$ thick membrane. This crosssectional view indicates that the fouling layer has a thickness of 16-29 $\mu \mathrm{m}$, and a very non-uniform spatial distribution. The $\beta$-D-glucopyranose polysaccharides were distributed over the fouling layer, while live cells were present as clusters in the layer. 

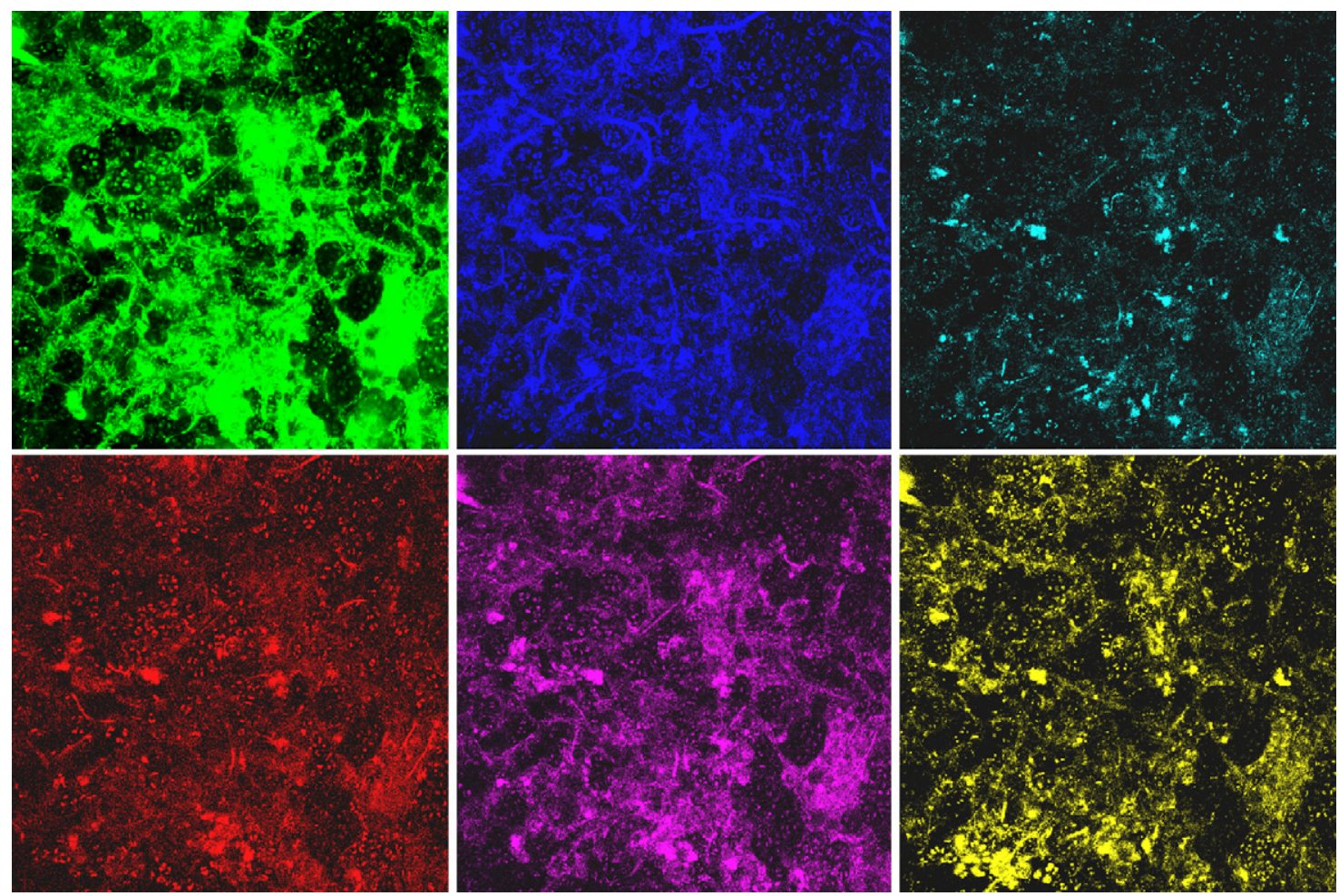

Fig. 1. CLSM images scanned at $5 \mu \mathrm{m}$ above membrane surface. Top view of fouling layer of image size $100 \mu \mathrm{m} \times 100 \mu \mathrm{m}$. Green: proteins, $\beta$-D-glucopyranose polysaccharides (blue), $\alpha$-D-glucopyranose polysaccharides (light blue), red: total cells, purple: dead cells, yellow: lipids. (For interpretation of the references to colour in this figure legend, the reader is referred to the web version of the article.)

\subsection{Building fouling layer model}

Using a series of CLSM images scanned on the fouling layer, the three dimensional distributions of nucleic acids and EPS

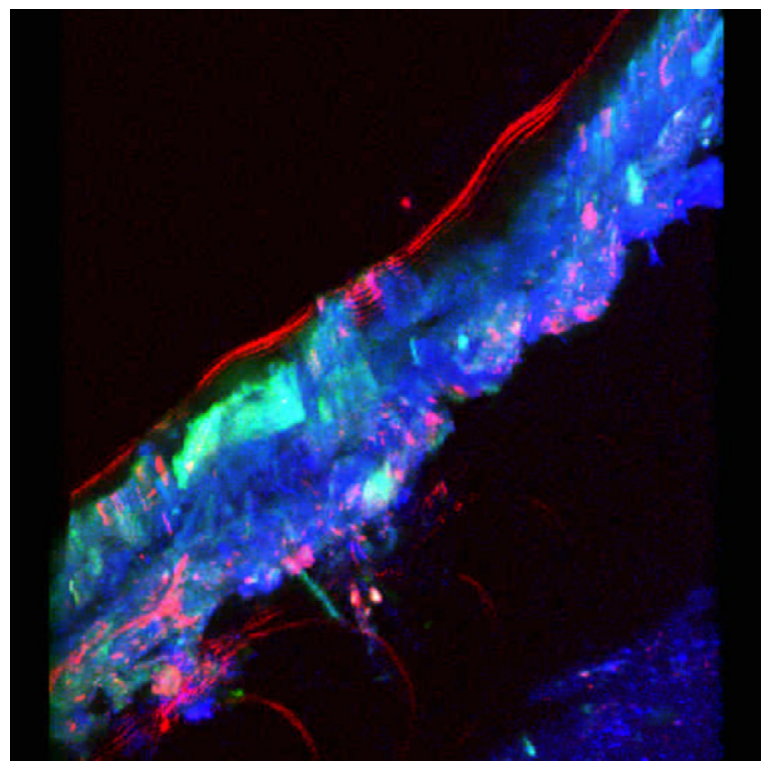

Fig. 2. Cross-sectional view of fouling layer combining the CLSM images of proteins (green), total cells (red), and $\beta$-D-glucopyranose polysaccharides (blue). The size of image is of length $100 \mu \mathrm{m}$. (For interpretation of the references to colour in this figure legend, the reader is referred to the web version of the article.) can be generated by a method presented elsewhere $[33,34]$. The CLSM image was bileveled with a threshold value determined by Otsu's method. Then, the boundaries in the bileveled images were classified by connectivity and whether they were in the object or its complement. The connectivity of neighboring pixels in all analyses was set to four to detect the edges. When the boundaries were sketched, the isosurfaces were developed to determine the object's three-dimensional shape. The advancingfront algorithm is adopted to fill each region that is defined by the simplified polygonal surface model of the pores, with unstructured tetrahedral volumetric grids [35]. Over $10^{6}$ grid cells were included in the mesh model to describe the interior of the fouling layer in Fig. 3 (the central, porous layer). The void volume divided by the total volume obtained yielded the mean porosity of the organic fouling layer, which was estimated at 0.49 .

The dark areas in the model represent the pore sites among the organic molecules, which were probed in the fouling layer. These can be blocked by other organic substances or inorganic particles. Accordingly, the flow field that was simulated using the fouling layer model in Fig. 3 represents the base case line to demonstrate the effects of blockage with additional inorganic particles on layer permeability. The mesh model of a fouling layer was placed perpendicular to an incoming Newtonian fluid of density $\rho$ and viscosity $\mu$, flowing at a uniform velocity of $V$ from left end far away from membrane surface. Fig. 3 also shows the boundaries of the flow process in the computation. 


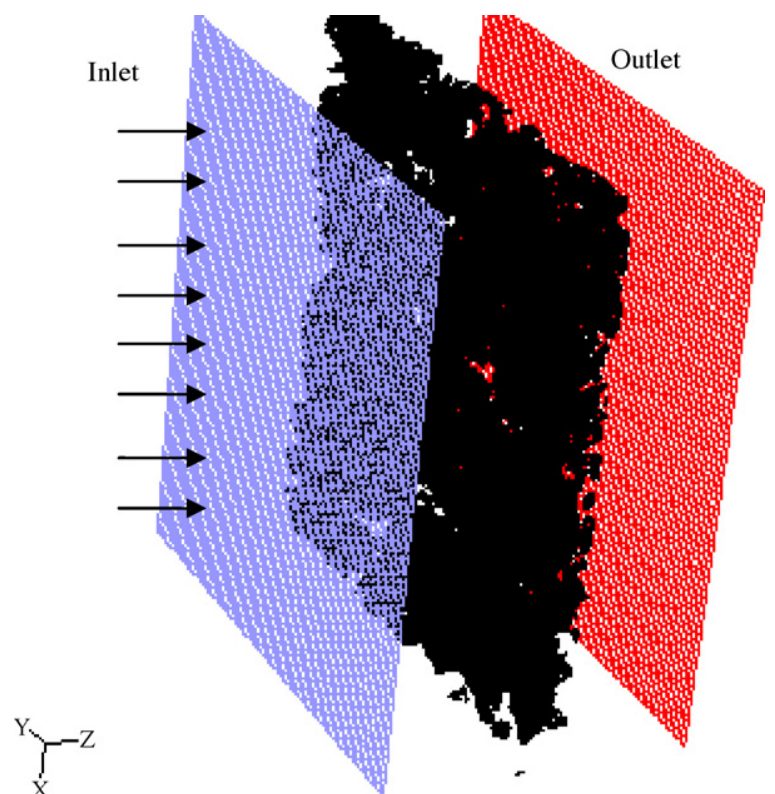

Fig. 3. The computational domain of the present fouling layer model. The approaching velocity $(V)$ is along the $z$-axis.

\subsection{Simulation of intra-layer flow field}

The steady-state Navior-Stokes equations are the governing equations of the intra-layer and surrounding flow velocity of $\vec{u}$,

$\left(\vec{u}^{\prime} \cdot \nabla\right) \vec{u}^{\prime}+\frac{P_{0}}{\rho V^{2}} \nabla P^{\prime}=\frac{1}{R e} \nabla^{2} \vec{u}^{\prime}$

where $\vec{u}^{\prime}=\vec{u} / V, P^{\prime}=P / P_{0}, R e=\rho H V / \mu ., P_{0}$ and $H$ are the characteristic pressure and length, respectively. The boundary conditions are as follows.

$\vec{u}=\vec{V} \quad$ at inlet surface

$\vec{u}=\overrightarrow{0} \quad$ at solid's surfaces of fouling layer

In the calculations, tetrahedral grid cells in the computational domain were initially preprocessed using geometrical modeling mesh generation software, GAMBIT 2.0 (Fluent, Inc., USA). Then, the governing equations (Eq. (1)) combined with boundary conditions (Eqs. (2a) and (2b)) were solved using FLUENT 6.1 software (Fluent, Inc., USA). The pressure-velocity coupling algorithm was SIMPLEC (Semi-Implicit Method for PressureLinked Equations-Consistent). The calculation was performed with a maximum relative error of $0.01 \%$.

Fig. 4 presents the contours of the cross-flow velocities over the planes 5, 10, 15 and $20 \mu \mathrm{m}$ above the membrane surface. A non-uniform flow pattern is observed. The area-weighted mean pressure of the cross sections perpendicular to the flow direction was adopted to determine the mean pressure drop in the floc. Permeability was estimated from Darcy's law. The permeability of the fouling layer was defined as [(superficial velocity $)($ viscosity $) /($ pressure gradient $)]=2.65 \times 10^{-12} \mathrm{~m}^{2}$.

The intrafloc flow pattern was displayed as a plot of the path lines. The trajectory function was defined as

$\frac{\mathrm{d} x}{\mathrm{~d} t}=u_{p, X}(x, y, z, t)$
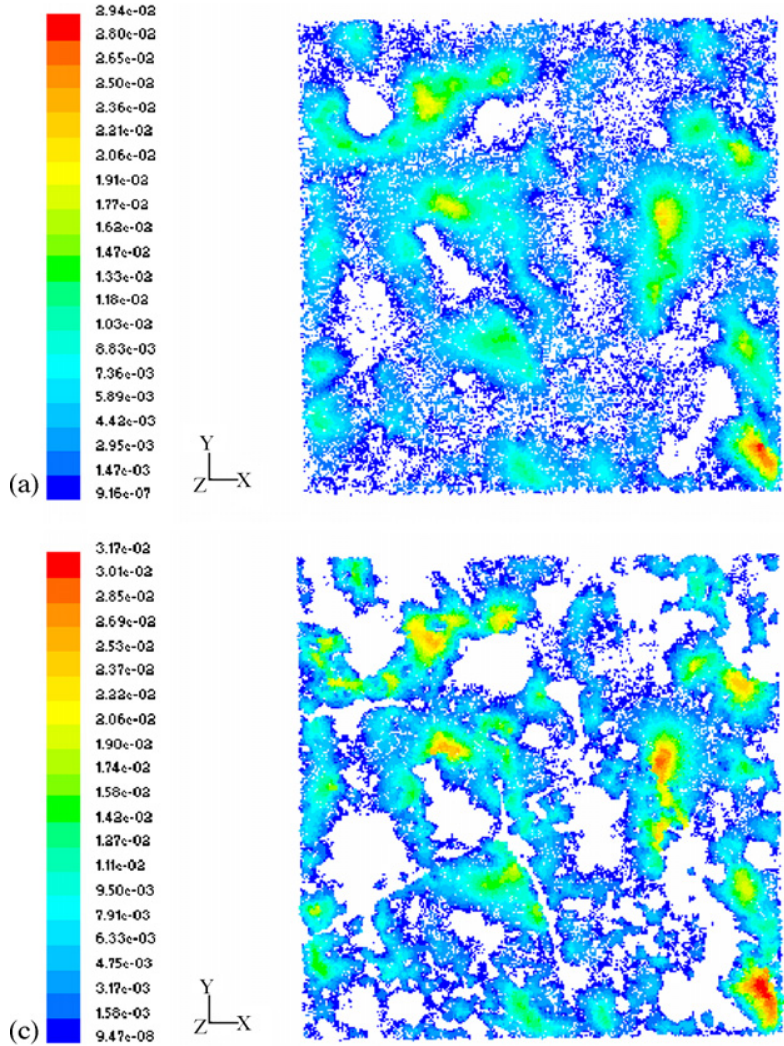
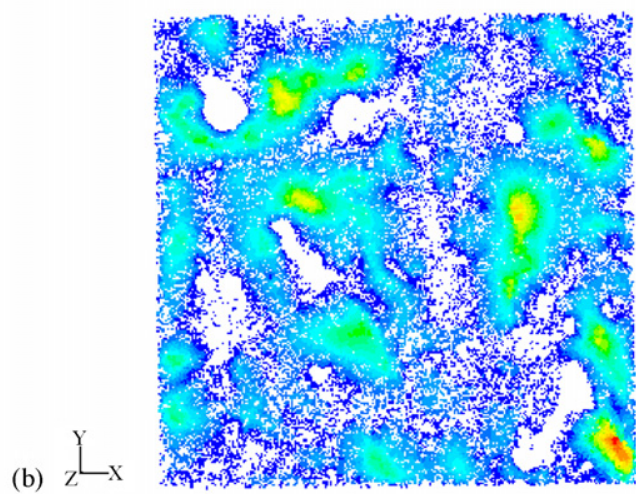

(d)

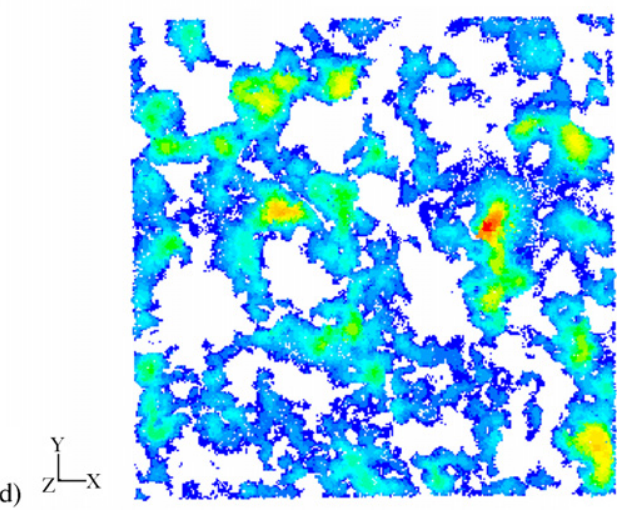

Fig. 4. Contours of upflow velocities in fouling layer. (a) $5 \mu \mathrm{m}$ from membrane surface; (b) $10 \mu \mathrm{m}$ from membrane surface; (c) $15 \mu \mathrm{m}$ from membrane surface; (d) $20 \mu \mathrm{m}$ from membrane surface. 


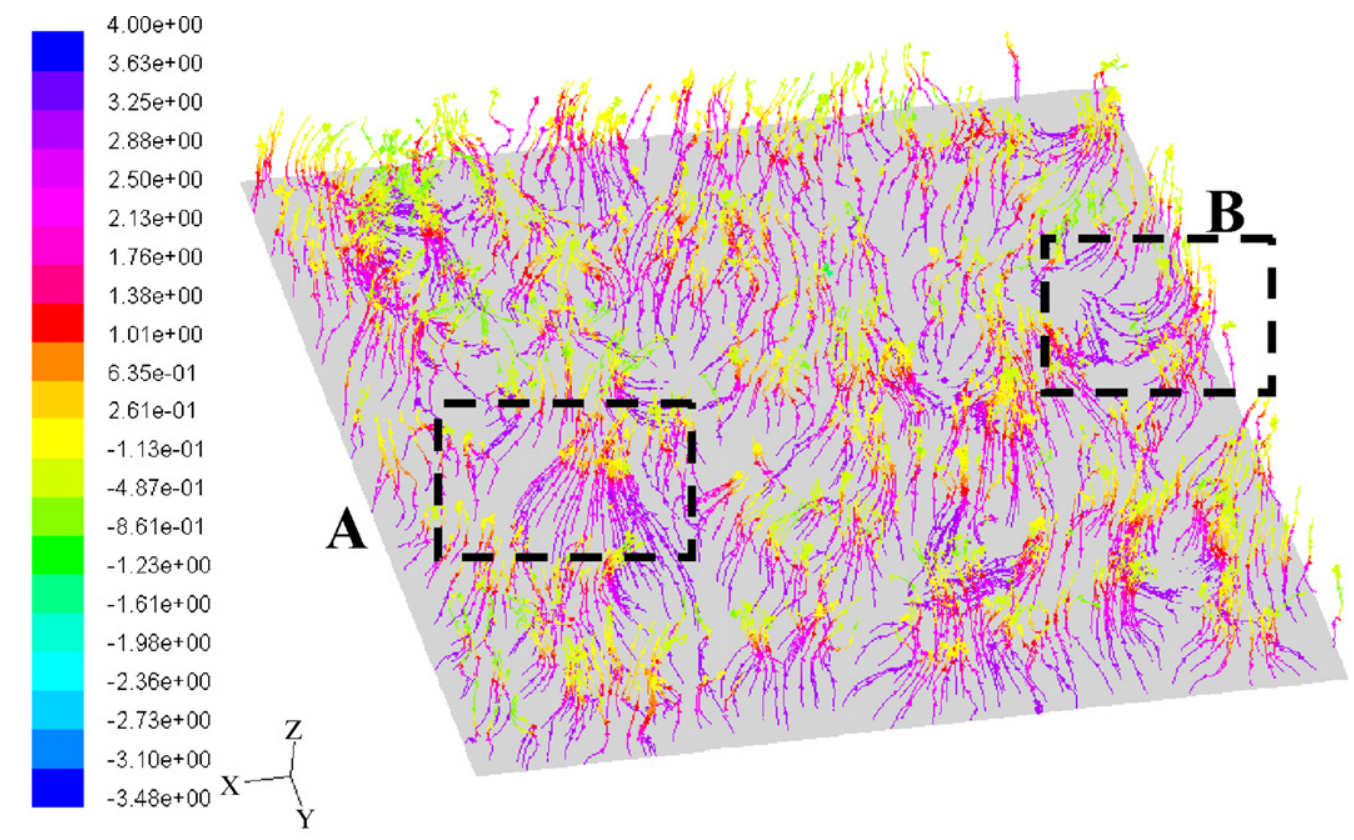

Path Lines Colored by Static Pressure (pascal)

May 12, 2006

FLUENT 6.1 (3d, segregated, lam)

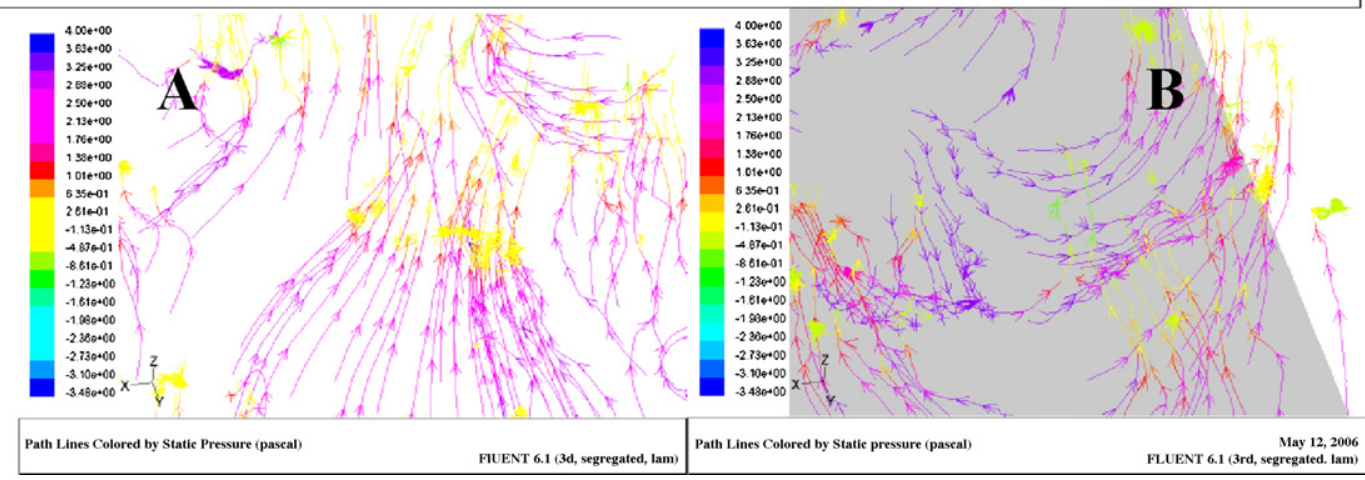

Fig. 5. Path lines calculated based on the investigated fouling layer model. Regions A and B are magnified to reveal detailed flow field.

$\frac{\mathrm{d} y}{\mathrm{~d} t}=u_{p, Y}(x, y, z, t)$

$\frac{\mathrm{d} z}{\mathrm{~d} t}=u_{p, Z}(x, y, z, t)$

Nine thousand fluid particles were initially released from the inlet plane and their paths through the fouling layer were individually monitored.

Fig. 5 plots the path lines, colored according to the static pressure (gauge), measured in Pa. A very complex flow pattern is noticeable. Magnified views of a section of the flow field (A and $\mathrm{B}$ in Fig. 5) reveal that, because of the inter-connectivity of the neighboring pores, the flow direction may even be the reverse of that of the pressure gradient. The typically adopted onedimensional fouling model is likely to be a poor approximation of the observed intra-layer flow field [32].

The path lines in Fig. 5 yield an estimate of channel tortuosity in the fouling layer. The path lengths along all lines were averaged, and the average was divided by the thickness of the layer. This ratio was defined as the mean tortuosity $\tau$, which was 1.42 for this fouling layer.

\subsection{Effects of pore blockage on permeability}

The known distributions of open pores over the layer must be used to determine how pore blockage affects the permeability of the fouling layer. When an image is transformed into a bileveled image, blobs (binary large objects) can be recognized and some features of the blobs, such as their areas and perimeters, can be quantified. The convex perimeter $\left(p_{\mathrm{C}, \mathrm{p}}\right)$ is an approximate perimeter of the convex hull of a blob derived from the eight Feret diameters of the blob. Chu and Lee [33] noted that the socalled the "maximum convex perimeter method" determines the threshold value to describe the spatial distribution of small pores or interstices among the solid blobs in a bioaggregate matrix. The cross-sectional area and volume of each pore are $A_{\mathrm{p}}$ and $V_{\mathrm{p}}$, respectively. Then, the shape of the pores is assumed to be circular so the pore diameter $d_{\mathrm{p}}$ is estimated as $\sqrt{\frac{4 A_{\mathrm{p}}}{\pi}}$. 

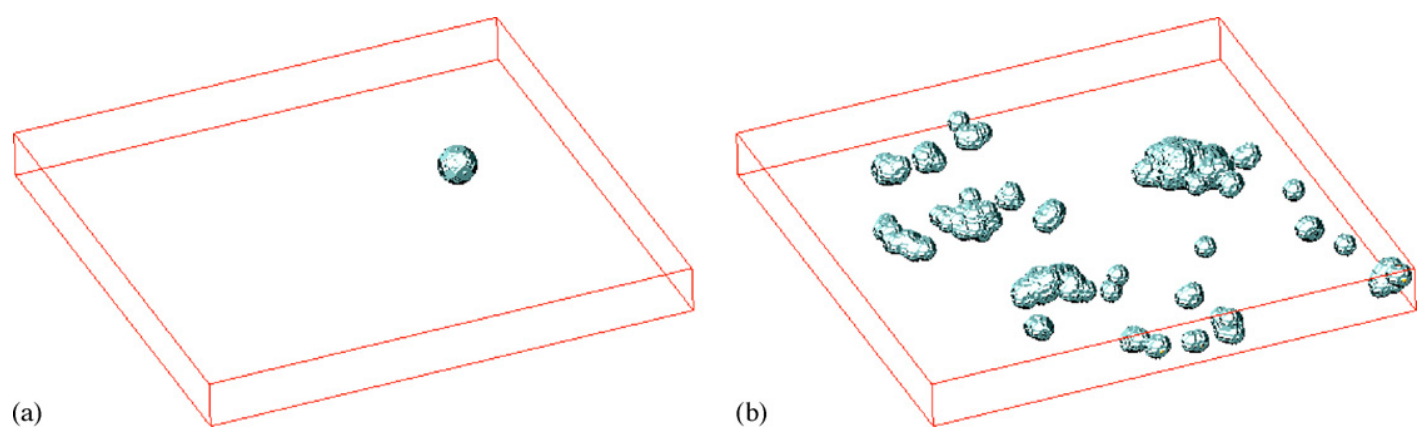

Fig. 6. The locations of internal pores inside the fouling layer. (a) Pore diameter $\geq 18.6 \mu \mathrm{m}$; (b) pore diameter $\geq 8.8 \mu \mathrm{m}$.

Only one pore exists with a diameter of over $18.6 \mu \mathrm{m}$ (Fig. 6a), but 42 pores have diameter $>8.8 \mu \mathrm{m}$ (Fig. 6b). Fig. 7 plots pore volume as a function of pore diameter. Pores with diameter $>8.8 \mu \mathrm{m}$ account for only about $15 \%$ of the porosity of the layer; the volume of the largest pore of size $18.6 \mu \mathrm{m}$ was $0.67 \%$ of the total volume of the pores.

The identified pores were blocked artificially and the obtained fouling layer models (with blocked pores) were reconstructed and the intra-layer flow fields were recalculated. Then the mean permeability of the fouling layer was estimated. Blocking the pores with a diameter of over $12.7 \mu \mathrm{m}$ reduces the porosity by $2.6 \%$; the permeability was reduced from $2.68 \times 10^{-12}$ to $2.26 \times 10^{-12} \mathrm{~m}^{2}-\mathrm{a} 15.6 \%$ reduction. Further blocking the pores of diameters $12.7-8.8 \mu \mathrm{m}$ reduced the permeability to $1.13 \times 10^{-12} \mathrm{~m}^{2}$, approximately by $58 \%$ of that of the unblocked biofouling layer. This observation is consistent with the note made by Wu et al. [36] that the largest pores in a porous medium are responsible for most of the advective flow.

Wu et al. [36] assumed that the bioaggregate interior could be modeled as a bundle of parallel straight round pipes, following the Hagen-Poiseuille law. That is, the mean permeability to which pores of size category $j$ contributes is,

$k_{j}=\frac{\varepsilon n_{j} d_{j}^{4}}{32 \sum_{1}^{N} n_{i} d_{i}^{2}}$

where the indices $i$ and $j$ are diameter category number $n_{1}$ that is, $i=1$ for $n_{1}$ pore diameter $>18.6 \mu \mathrm{m} ; i=2$ for $n_{2}$ diameters from

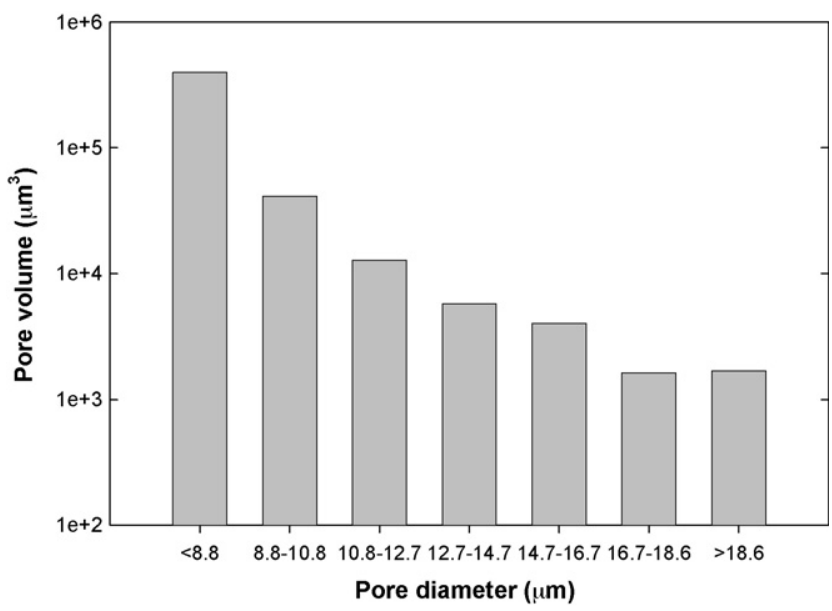

Fig. 7. The volume vs. diameter of internal pores inside the fouling layer.
16.7 to $18.6 \mu \mathrm{m}$, and so on. The permeability associated with the individual pores in category $j$ satisfies $\left(k_{j} / n_{j}\right) \propto d_{j}^{4}$. The parameter $k_{j}$ of the pores in each category can be determined from Fig. 8, and the number of pores can be estimated from the pore volume and the pore thickness, based on the assumption that the pores are cylindrical pipes. Fig. 9 shows that the calculated $\left(k_{j} / n_{j}\right)$ value is approximated by a power-law in $d_{j}^{4}$. Therefore, the assumption that the interior pores can be treated as a bundle of parallel pipes is basically correct. However, the simulation results that are based on a complex structure (Fig. 3) reveal a

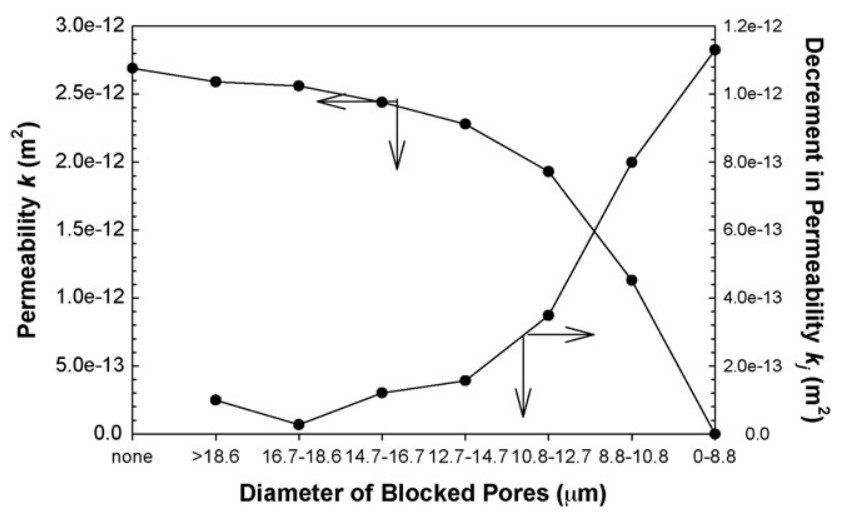

Fig. 8. The permeability of fouling layer and decrement in permeability vs. diameters of blocked pores inside fouling layer.

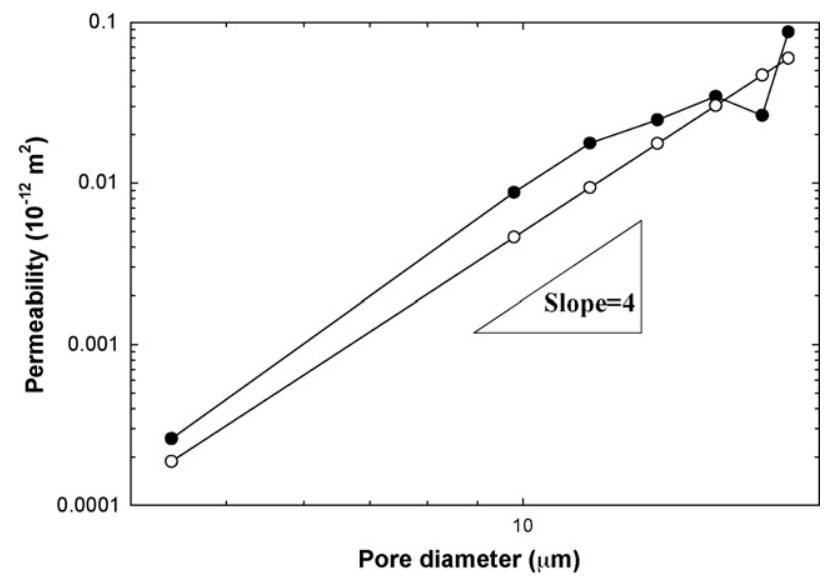

Fig. 9. The permeability contributed by each pore in fouling layer vs. pore diameter plot. Solid symbols: data from Fig. 8; open symbols: calculation based on Eq. (4). 
higher permeability than is predicted by the Hagen-Poiseuille law (Eq. (4)), perhaps because many intersects among various pores facilitate advection flow when some pores are blocked.

\section{Conclusions}

Biofouling of membrane limits the performance of numerous membrane separation processes. The intra-layer flow fields, yielded by the applied pressure difference and the detailed structure of the biofouling layer, controls the permeate flow rates. The supernatant of an MBR sludge was filtered in dead-end mode with a mixed cellulose ester membrane. The structure of the formed biofouling layer was probed using a six-fold staining scheme combined with CLSM imaging technique, yielding the three-dimensional distributions of nucleic acids, proteins, $\alpha$-D-glucopyranose polysaccharides, $\beta$ D-glucopyranose polysaccharides and lipids in the fouling layer. Imaging processing produced the corresponding volumetric grid model of the fouling layer, which was used to simulate the intra-layer flow field during filtration. With the built model, the effective permeability of the fouling layer was estimated and the effects of pore blockage inside the layer were illustrated. The demonstrated scheme could be applicable to various membrane separation processes with biofouling, with a potential use to understand how biofouling layer affects performance of a running MBR system.

\section{References}

[1] A. Ramesh, D.J. Lee, M.L. Wang, J.P. Hsu, R.S. Juang, K.J. Hwang, J.C. Liu, S.J. Tseng, Biofouling in membrane bioreactor, Sep. Sci. Technol. 41 (2006) 1345-1370.

[2] P. Le-Clech, V. Chen, A.G. Fane, Fouling in membrane bioreactors used in wastewater treatment-a review, J. Membr. Sci. 284 (2006) 17-53.

[3] K. Kimura, N. Yamato, H. Yamamura, Y. Watanabe, Membrane fouling in pilot-scale membrane bioreactors (MBR2) treating municipal wastewater, Environ. Sci. Technol. 39 (2005) 6293-6299.

[4] H. Nagaoka, S. Yamanishi, A. Miya, Modeling of biofouling by extracellular polymers in a membrane separation activated sludge system, Water Sci. Technol. 38 (1998) 497-504.

[5] H. Nagaoka, S. Ueda, A. Miya, Influence of bacterial extracellular polymers on the membrane separation activated sludge process, Water Sci. Technol. 34 (1996) 165-172.

[6] G.L. Leslie, R.P. Schneider, A.G. Fane, K.C. Marshall, C.J.D. Fell, Fouling of a microfiltration membrane by two Gram-negative bacteria, Colloids Surf. A 73 (1993) 165-178.

[7] G.G. Geesey, M.W. Stupy, P.J. Bremer, The dynamics of biofilms, Int. Biodetor. Biodegrad. 30 (1992) 135-154.

[8] I.S. Chang, P. Le-Clech, B. Jefferson, S.J. Judd, Membrane fouling in membrane bioreactors for wastewater treatment, J. Environ. Eng. 128 (2002) 1018-1029.

[9] B.D. Cho, A.G. Fane, Fouling transients in nominally sub-critical flux operation of a membrane bioreactor, J. Membr. Sci. 209 (2002) 391403.

[10] I.S. Chang, C.H. Lee, Membrane filtration characteristics in membranecoupled activated sludge systems-the effect of physiological states of activated sludge on membrane fouling, Desalination 120 (1998) 221-233.

[11] S. Rosenberger, M. Kraume, Filterability of activated sludge in membrane bioreactor, Desalination 146 (2002) 373-379.

[12] M.E.H. Rojas, M.R. van Kaam, S. Schetrite, C. Albasim, Role and variations of supernatant compounds in submerged membrane bioreactor fouling, Desalination 179 (2005) 95-107.
[13] W. Lee, S. Kang, H.S. Shin, Sludge characteristics and their contribution to microfiltration in submerged membrane bioreactors, J. Membr. Sci. 216 (2003) 217-227.

[14] E.H. Bouhabila, R. Ben Aim, H. Buisson, Fouling characterization in membrane bioreactors, Sep. Purif. Technol. 22-23 (2001) 123-132.

[15] C. Wisniewski, A. Grasmick, Floc size distribution in a membrane bioreactor and consequences for membrane fouling, Colloids Surf. A 138 (1998) 403-411.

[16] J.M. Lee, W.Y. Ahn, C.H. Lee, Comparison of the filtration characteristics between attached and suspended growth microorganisms in submerged membrane bioreactor, Water Res. 35 (2001) 2435-2445.

[17] J.W. Cho, K.G. Song, S.H. Lee, K.H. Ahn, Sequencing anoxic/anaerobic membrane bioreactor (SAM) pilot plant for advanced wastewater treatment, Desalination 178 (2005) 219-225.

[18] K. Stamatakis, C. Tien, Cake formation and growth in cake filtration, Chem. Eng. Sci. 46 (1991) 1917-1933.

[19] M.A. Koenders, R.J. Wakeman, The initial stages of compact formation from suspensions by filtration, Chem. Eng. Sci. 51 (1996) 3897-3908.

[20] B. Jefferson, A. Brookes, P. Le-Clech, S.J. Judd, Methods for understanding organic fouing in MBRs, Waste Sci. Technol. 49 (2004) 237-244.

[21] A. Brookes, S.J. Judd, E. Reid, E. Germain, S. Smith, H. Alvarez-Vazquez, P. Le-Clech, T. Stephenson, E. Turra, B. Jefferson, Biomass characterization in membrane bioreactors, in: Proceedings of IMSTEC, Sydney, Australia, 2003.

[22] H. Nakaoka, H. Nemoto, Influence of extracellular polymeric substances on nitrogen removal in an intermittently aerated membrane bioreactor, Waste Sci. Technol. 51 (2005) 151-158.

[23] B. Jesjean, S. Rosenberfer, C. Laabs, M. Jekel, R. Gnirss, G. Amy, Correlation between membrane fouling and soluble/colloidal organic substances in membrane bioreactors for municipal wastewater treatment, Water Sci. Technol. 51 (2005) 1-8.

[24] S. Rosenberger, H. Evenblij, S. te Poele, T. Wintgens, C. Laabs, The importance of liquid phase analyses to understand fouling in membrane assisted activated sludge processes-six case studies of different European research groups, J. Membr. Sci. 263 (2005) 113-126.

[25] H. Evenblij, S. Geilvoet, J. Van der Graaf, H.F. Van der Roest, Filtration characterisation for assessing MBR performance: three cases compared, Desalination 178 (2005) 115-124.

[26] K. Tarnacki, S. Lyko, T. Wintgens, T. Melin, F. Natau, Impact of extracellular polymeric substances on the filterability of activated sludge in membrane bioreactors for landfill treatment, Desalination 179 (2005) 181190.

[27] T.R. Neu, J.R. Lawrence, Lectin-binding analysis in biofilm system, Method Enzymol. 310 (1999) 145-152.

[28] M. Strathmann, J. Wingender, H.C. Flemming, Application of fluorescently labeled lectins for the visualization and biochemical characterization of polysaccharides in biofilms of Pseudomonas Aeruginosa, J. Microbiol. Meth. 50 (2002) 237-248.

[29] M.A. Yun, K.M. Yeon, J.S. Park, C.H. Lee, J. Chun, D.J. Lim, Characterization of biofilm structure and its effect on membrane permeability in MBR for dye wastewater treatment, Water Res. 40 (2006) 45-52.

[30] H.Y. Kim, K.M. Yeon, C.H. Lee, S.H. Lee, T. Swaminathan, Biofilm structure and extracellular polymeric substances in low and high oxygen bioreactors, Sep. Sci. Technol. 41 (2006) 1213-1230.

[31] M.Y. Chen, D.J. Lee, J.Y. Lai, Excellular polymeric substances in fouling layer, Sep. Sci. Technol. 41 (2006) 1345-1370.

[32] M.Y. Chen, D.J. Lee, Z. Yang, X.F. Peng, J.Y. Lai, Fluorecent staining for study of extracellular polymeric substances in membrane biofouling layers, Environ. Sci. Technol. 40 (2006) 6642-6646.

[33] C.P. Chu, D.J. Lee, Bilevel thresholding of image of sludge flocs, Environ. Sci. Technol. 38 (2004) 1161-1169.

[34] C.P. Chu, D.J. Lee, Effects of pore geometry on intrafloc flow, J. Chem. Inst. Chem. Eng. 35 (2004) 659-666.

[35] C.P. Chu, D.J. Lee, Multiscale structure of biological flocs, Chem. Eng. Sci. 59 (2004) 1875-1883.

[36] R.M. Wu, D.J. Lee, P.J. He, Estimation of floc permeability and porosity, J. Chin. Inst. Chem. Eng. 34 (2003) 275-280. 